\title{
Clinical Efficacy of Intra-Articular Injection With P- PRP Versus That of L-PRP in Treating Knee Cartilage Lesion: A Randomized Controlled Trial.
}

Yiqin Zhou

Shanghai Changzheng Hospital

Haobo Li

Shanghai Changzheng Hospital

Shiqi Cao

Chinese PLA General Hospital

Yaguang Han

Shanghai Changzheng Hospital

Jiahua Shao

Shanghai Changzheng Hospital

Qiwei Fu

Shanghai Changzheng Hospital

\section{Bo Wang}

Shanghai Changzheng Hospital

Jun Wu

Shanghai Changzheng Hospital

Dong Xiang

Shanghai Changzheng Hospital

Ziye Liu

Shanghai Changzheng Hospital

Huan Wang

Shanghai Changzheng Hospital

Jun Zhu

Shanghai Changzheng Hospital

Qirong Qian ( $\sim$ qianqr@163.com )

Second Military Medical University

Xiaolei Yang

Shanghai Changzheng Hospital

\section{Song Wang}

Shanghai Changzheng Hospital 
Research article

Keywords: Knee cartilage lesion, Randomized controlled trial, P-PRP, L-PRP, Cartilage regeneration

Posted Date: September 7th, 2021

DOl: https://doi.org/10.21203/rs.3.rs-864698/v1

License: (c) (i) This work is licensed under a Creative Commons Attribution 4.0 International License. Read Full License 


\section{Abstract}

Background: To evaluate the clinical efficacy and safety of intra-articular injection with pure platelet-rich plasma (P-PRP) versus those of leukocyte platelet-rich plasma (L-PRP) in treating knee cartilage lesions, we conducted a double-blind, randomized controlled clinical trial with a larger sample and longer followup period.

Methods: From October 2019 to October 2020, 95 patients were invited to participate in our study, and 60 (63.2\%) were randomized to P-PRP $(n=30)$ or L-PRP $(n=30)$ groups. Patients from the two groups were treated with knee intra-articular injections of P-PRP or L-PRP. Visual analog scale (VAS) and Western Ontario and McMaster Universities Arthritis Index (WOMAC) scores were assessed preoperatively and at 6 weeks, 12 weeks, 6 months, and 12 months after intervention.

Results: We followed up 27 cases in the P-PRP group and 26 cases in the L-PRP group. No significant differences in VAS and WOMAC scores were found between the two groups before the intervention $(P>0.05)$. The WOMAC Pain and VAS-Motions scores of the P-PRP group were significantly lower than those of the L-PRP group at 6 weeks after the intervention $(P<0.05)$. While the long-term clinical efficacy of both injections was similar and weakened after 12 months, more adverse events were found in the LPRP group.

Conclusions: The short-term results of our study are encouraging and demonstrate that both L-PRP and P-PRP intra-articular injections reduce pain and improve function in patients with knee cartilage lesions. Compared with the L-PRP injection, the P-PRP injection (which had a lower risk of early inflammation caused by leukocytes) showed better clinical efficacy in the early phase of postoperative rehabilitation and resulted in fewer adverse events. However, long-term clinical efficacy for both injections were similar and weakened after 12 months.

Trial registration: ChiCTR1900026365. Registered on 3 October 2019, http://www.chictr.org.cn/showproj.aspx?proj=43911.

\section{Background}

Articular cartilage lesions of the knee are extremely common, with a high morbidity rate of $5 \%$ in the general population and are characterized by the progressive loss of joint function and development of pain [1-4]. Currently, traditional conservative treatments for knee cartilage lesions include nonsteroidal anti-inflammatory drugs, glucosamine, hyaluronic acid, and glucocorticoids [5-8]. However, as these therapies have limited therapeutic efficacy in the healing of cartilage lesions, their long-term efficacy is uncertain [9]. In addition, while these known therapies can be effective for a short period, they can cause several adverse reactions [10].

Platelet-rich plasma (PRP) is a blood derivative derived from autogenous whole blood containing growth factors and cytokines [11-13]. In the past few years, the application of PRP in the regeneration of 
cartilage lesions has proven to be simple, low-cost, and less invasive [14-19]. Initial studies have primarily demonstrated the feasibility and safety of PRP injection into the knee articular cavity for treating cartilage lesions, but several subsequent studies reported controversial therapeutic efficacy associated with the component of PRP, especially the different concentrations of leukocytes $[12,20,21]$.

Based on the concentration of leukocytes, PRP can be divided into two types: pure PRP (P-PRP) and leukocyte PRP (L-PRP) [22]. In recent years, studies have shown that inflammatory factors, such as interleukin $1 \beta$ (IL-1 $\beta)$ and tumor necrosis factor- $a$ (TNF-a), are associated with the concentration of leukocytes in PRP [23-25]. Since these inflammatory factors may have a negative effect on cartilage regeneration, some studies showed a better treatment efficacy of P-PRP compared with that of L-PRP. On the other hand, other researchers have indicated that L-PRP, with the greater release of cytokines and enzymes from leukocytes and less loss of purification of PRP, has equal therapeutic efficacy as P-PRP in the repair of cartilage lesions $[22,26,27]$. Since leukocytes are a beneficial source of cytokines and enzymes, they may be important for the prevention of infection [28-32]. For further clinical studies with more patients, longer follow-up periods, and higher evidence, we conducted a randomized controlled trial (RCT) to evaluate the therapeutic efficacy of P-PRP versus that of L-PRP in treating knee cartilage lesions.

\section{Methods}

\section{Trial design}

This double-blind, RCT of Evidence Level I compared the safety and efficacy of P-PRP with those of LPRP in Chinese patients with knee cartilage lesions. This clinical trial was registered at www.chictr.org.cn (ChiCTR1900026365), approved by the Clinical Research Ethics Committees of the hospital of the authors [CZEC (2017)-04], and followed the ethical standards of the institutional and national research committees and the 1964 Helsinki Declaration and its later amendments. Informed consent was obtained from all the participants in the study. From October 2019 to October 2020, 60 patients with knee cartilage lesions were recruited to participate in this study the hospital of the authors.

\section{Trial participants}

The following inclusion criteria were used for patient selection: 1. Patients between the ages of 18 and 75 years. MRI clearly indicated articular cartilage injury, and the Kellgren-Lawrence standard knee grade was not more than level-3. 3. Patients with obvious knee pain or discomfort lasting for more than 3 months. 4 . Patients who were willing to participate in the study and signed the informed consent form. 5. Patients who had articular cartilage injury diagnosed by arthroscopy and who did not receive targeted treatment.

Patients with the following characteristics were excluded from the study: 1 . Other surgical procedures were performed to treat the articular cartilage. 2. Patients who had a history of intra-articular injection or peri-articular invasive treatments and procedures within 3 months. 3 . Symptoms and imaging findings that were localized in the patellofemoral joint. 4. Patients who suffered from malignant neoplasms. 5. Patients who had active infection in other parts of the body. 6 . Female patients who were pregnant, 
lactating, or preparing for pregnancy. 7. Cartilage lesions caused by infectious or gouty arthritis. 8 . Patients who had autoimmune diseases such as rheumatoid arthritis and ankylosing spondylitis. 9. Patients who had diabetes with FBG over $8 \mathrm{mmol} / \mathrm{L}$ and had poor control. 10. Patients who were generally in poor condition and unable to tolerate surgery. 11. Patients who had severe diseases such as cerebral hemorrhage, severe pneumonia, and multiple organ dysfunction. 12. Patients with Charcot joint. 13. Any conditions that might increase the patient's risk or influence the results of the experiment during the research. 14 . Other reasons making the patient unsuitable for this study.

Demographic data of sex, age, BMI, side of affected joint, and knee cartilage lesion stage was recorded after the patients were included in this study (Table 1).

Table 1

Baseline Characteristics of the Patients.

\begin{tabular}{|llll|}
\hline Characteristic & P-PRP group & L-PRP group & P \\
\hline Cases(Complete F-U) & $30(27)$ & $30(26)$ & \\
\hline Age & $62.27 \pm 5.27$ & $61.77 \pm 6.25$ & $>0.05$ \\
\hline Gender(M/F) & 7,23 & 9,21 & $>0.05$ \\
\hline BMI(Kg/M2) & $25.45 \pm 2.44$ & $25.61 \pm 4.04$ & $>0.05$ \\
\hline Whole blood count $\left(\times 10^{9} / \mathrm{L}\right)$ & & & \\
\hline Platelet & $166.32 \pm 58.55$ & $156.73 \pm 47.63$ & $>0.05$ \\
\hline WBC & $6.41 \pm 2.78$ & $6.89 \pm 3.42$ & $>0.05$ \\
\hline
\end{tabular}

\section{Randomization and blinding}

Patients who met the inclusion and exclusion criteria were randomly assigned into the following two groups using a block of three randomizations with computer-generated random numbers

(http://www.randomizer.org/) by specific grouping staff: P-PRP group (Group 1, $\mathrm{n}=30$ ) and L-PRP group (Group 2, $n=30$ ). The patients' group allocation was recorded in a secure dataset (Microsoft Office Excel 2013) and blinded to all patients and statisticians involved in the study. To ensure blinding, the following regulations were strictly executed: (1) The grouping staff was a statistician not involved in the treatment, evaluation, follow-up, and data analysis of the included patients. (2) Before PRP preparation, $50 \mathrm{~mL}$ of blood was obtained from each patient, numbered in sequence, and transferred to the laboratory. The type of PRP for a specific blood sample was then assigned according to the patients' group allocation by grouping staff. (3) Researchers who prepared the PRP only knew the number and type of PRP in the blood sample but did not know the corresponding patient's information. (4) After PRP preparation, $5 \mathrm{~mL}$ of P- or L-PRP was obtained, marked in its original number without its PRP type, and injected into the corresponding patient's knee joint. (5) Intra-articular injection of P-PRP or L-PRP was performed following 
the same para-patellar tendon approach without additional, distinguished incisions. (6) During statistical analysis, information of the patients' identity was hidden to statisticians, and grouping information was presented as groups 1 and 2 without the type of PRP.

\section{Study design}

\section{Preparation for L-PRP and P-PRP}

\section{L-PRP}

The procedure consisted of a 40-ml venous blood sample for every knee treated. PRP preparation kits (VEGO, China) were used to acquire the L-PRP. Two centrifugations (the first at 1,800 rpm for $15 \mathrm{~min}$ to separate erythrocytes by collecting the platelet-rich supernatant and the middle buffy coat above the red blood cell pellet in each tube, and a second at 3,500 rpm for $10 \mathrm{~min}$ to concentrate platelets) produced 5 $\mathrm{ml}$ of L-PRP. The concentrations of platelets and WBCs were tested using a blood analyzer. All open procedures were performed in an A-class sterile hood. Injections were administered 3 times every 14 days. This procedure was repeated for every injection. (Fig. 1)

\section{P-PRP}

The procedure consisted of a 50-ml venous blood sample for every knee treated. PRP preparation kits (VEGO, China) were used to acquire P-PRP. Two centrifugations (the first at 1,800 rpm for $15 \mathrm{~min}$ to separate erythrocytes and WBC by collecting the platelet-rich supernatant without the middle buffy coat above the red blood cell pellet in each tube, carefully avoiding harvesting of leukocytes, and a second at 3,500 rpm for $10 \mathrm{~min}$ to concentrate platelets) produced $5 \mathrm{ml}$ of P-PRP. The concentrations of platelets and WBCs were tested using a blood analyzer. All open procedures were performed in an A-class sterile hood. Injections were administered 3 times every 14 days. This procedure was repeated for every injection. (Fig. 1)

\section{Post-injection care and outcome measures}

After each injection, the patient was sent home with instructions to limit the use of the leg for at least 24 $\mathrm{h}$ and to use cold therapy/ice on the affected area for pain. During this period, the use of nonsteroidal medications was prohibited. During the treatment period, rest or mild activities (such as an exercise bike and mild exercises in the pool) were indicated; subsequently, the gradual resumption of normal sports or recreational activities was allowed as tolerated. Complications and adverse events were also recorded.

Knee function and health-related quality of life of the included patients in this trial were evaluated using the visual analog scale (VAS) and Western Ontario and McMaster Universities Osteoarthritis Index (WOMAC) measured at baseline and at 6 weeks, 12 weeks, 6 months, and 12 months after the last treatment.

\section{Statistical analysis}


Statistical analysis was performed using the Statistical Package for the Social Sciences (SPSS Inc., version 20.0). Chicago, IL). Differences were considered significant at two-sided $p$ values $<0.05$.

The normality of the numerical data was identified using the Shapiro-Wilk normality test and Q-Q graphs. Metric variables with normal distribution were presented as mean \pm standard deviation and analyzed using an unpaired t-test for independent samples. Non-Gaussian variables were analyzed using the Friedman test. Categorical variables were analyzed using Chi-square and Fisher's exact tests. Because the primary outcome was the difference in WOMAC scores between baseline and 12 months, the sample size was set based on the results of a previous study (a risk 0.05 , power $0.8,10 \%$ losses to follow-up, changes in WOMAC score 16.6, and SD 8.2). The required number of patients was 30.

\section{Results}

\section{Participants and baseline characteristics}

From October 2019 to October 2020, a total of 95 patients were invited to participate in our study, and 60 (63.2\%) were randomized to P-PRP $(n=30)$ or L-PRP $(n=30)$ groups, respectively (Fig. 2). All included patients in both P-and L-PRP group completed the 2-week treatment process, and all patients completed the final 1-year follow-up without withdrawal. Detailed demographic and clinical characteristics of participants from the two groups were comparable and listed in Table 1. The concentration of platelet in P-PRP was tested to be $486.71 \pm 65.75 \times 10^{9} / \mathrm{L}$ while that of L-PRP was $577.83 \pm 71.76 \times 10^{9} / \mathrm{L}$. The concentration of leukocytes in P-PRP was tested to be $0.05 \pm 0.03 \times 10^{9} / \mathrm{L}$ while that of L-PRP was $8.25 \pm$ $2.41 \times 10^{9} / \mathrm{L}$.

\section{Clinical outcomes}

Clinical outcomes for all patients were evaluated by VAS and WOMAC score preoperatively, and 6 weeks, 3 months, 6 months and 1 year after the last injection, which were listed in Table 2. 
Table 2

Outcome measures at pre-op, 6 weeks, 12 weeks, 6 months and 12 months

\begin{tabular}{|c|c|c|c|c|c|c|}
\hline Characteristic & Group & Pre-Op & 6 Weeks & 12 Weeks & 6 Months & 12 Months \\
\hline \multirow[t]{3}{*}{ WOMAC pain } & $\begin{array}{l}\text { P- } \\
\text { PRP }\end{array}$ & $7.77 \pm 4.04$ & $4.40 \pm 2.80$ & $3.60 \pm 2.84$ & $3.37 \pm 1.77$ & $5.80 \pm 2.85$ \\
\hline & L-PRP & $7.70 \pm 3.42$ & $5.87 \pm 2.99$ & $4.17 \pm 3.41$ & $3.50 \pm 2.36$ & $5.87 \pm 3.16$ \\
\hline & $\mathrm{p}$ & $>0.05$ & $<0.05$ & $>0.05$ & $>0.05$ & $>0.05$ \\
\hline \multirow[t]{3}{*}{$\begin{array}{l}\text { WOMAC } \\
\text { stiffness }\end{array}$} & $\begin{array}{l}\text { P- } \\
\text { PRP }\end{array}$ & $4.13 \pm 1.36$ & $3.60 \pm 1.04$ & $3.20 \pm 1.45$ & $3.17 \pm 1.66$ & $3.87 \pm 1.93$ \\
\hline & L-PRP & $4.27 \pm 1.48$ & $3.43 \pm 1.55$ & $3.23 \pm 1.25$ & $3.20 \pm 1.42$ & $3.93 \pm 1.46$ \\
\hline & $\mathrm{p}$ & $>0.05$ & $>0.05$ & $>0.05$ & $>0.05$ & $>0.05$ \\
\hline \multirow[t]{3}{*}{$\begin{array}{l}\text { WOMAC } \\
\text { function }\end{array}$} & $\begin{array}{l}\text { P- } \\
\text { PRP }\end{array}$ & $\begin{array}{l}24.47 \pm \\
12,94\end{array}$ & $\begin{array}{l}14.83 \pm \\
10.16\end{array}$ & $\begin{array}{l}13.93 \pm \\
10.74\end{array}$ & $\begin{array}{l}13.10 \pm \\
10.19\end{array}$ & $\begin{array}{l}22.07 \pm \\
10.45\end{array}$ \\
\hline & L-PRP & $\begin{array}{l}24.27 \pm \\
11.22\end{array}$ & $\begin{array}{l}16.37 \pm \\
12.20\end{array}$ & $\begin{array}{l}14.90 \pm \\
9.81\end{array}$ & $\begin{array}{l}13.27 \pm \\
9.63\end{array}$ & $\begin{array}{l}22.37 \pm \\
9.50\end{array}$ \\
\hline & $\mathrm{p}$ & $>0.05$ & $>0.05$ & $>0.05$ & $>0.05$ & $>0.05$ \\
\hline \multirow[t]{3}{*}{ WOMAC total } & $\begin{array}{l}\text { P- } \\
\text { PRP }\end{array}$ & $\begin{array}{l}37.37 \pm \\
13.84\end{array}$ & $\begin{array}{l}22.83 \pm \\
10.85\end{array}$ & $\begin{array}{l}20.73 \pm \\
11.42\end{array}$ & $\begin{array}{l}19.63 \pm \\
10.15\end{array}$ & $\begin{array}{l}31.73 \pm \\
10.50\end{array}$ \\
\hline & L-PRP & $\begin{array}{l}36.23 \pm \\
12.56\end{array}$ & $\begin{array}{l}25.67 \pm \\
13.32\end{array}$ & $\begin{array}{l}22.30 \pm \\
11.02\end{array}$ & $\begin{array}{l}19.97 \pm \\
9.74\end{array}$ & $\begin{array}{l}32.17 \pm \\
10.82\end{array}$ \\
\hline & $\mathrm{p}$ & $>0.05$ & $>0.05$ & $>0.05$ & $>0.05$ & $>0.05$ \\
\hline \multirow[t]{3}{*}{ VAS-Static } & $\begin{array}{l}\text { P- } \\
\text { PRP }\end{array}$ & $1.87 \pm 1.31$ & $1.43 \pm 1.04$ & $1.37 \pm 0.81$ & $1.57 \pm 1.04$ & $1.63 \pm 0.85$ \\
\hline & L-PRP & $1.93 \pm 1.08$ & $1.67 \pm 0.99$ & $1.43 \pm 1.01$ & $1.60 \pm 1.07$ & $1.77 \pm 0.90$ \\
\hline & $\mathrm{p}$ & $>0.05$ & $>0.05$ & $>0.05$ & $>0.05$ & $>0.05$ \\
\hline \multirow[t]{3}{*}{ VAS-Motion } & $\begin{array}{l}\text { P- } \\
\text { PRP }\end{array}$ & $5.77 \pm 1.79$ & $3.07 \pm 1.48$ & $2.87 \pm 1.43$ & $2.60 \pm 1.19$ & $5.30 \pm 1.02$ \\
\hline & L-PRP & $5.80 \pm 1.85$ & $4.20 \pm 1.32$ & $3.17 \pm 1.60$ & $2.83 \pm 1.15$ & $5.37 \pm 1.38$ \\
\hline & $\mathrm{p}$ & $>0.05$ & $<0.01$ & $>0.05$ & $>0.05$ & $>0.05$ \\
\hline
\end{tabular}

\section{VAS score}

Pain of patients, evaluated by VAS static and motion, showed similar trends in both P- and L-PRP group. For VAS static, comparing to baseline level, value of VAS static in P-PRP group improved from $1.87 \pm 1.31$ to $1.43 \pm 1.04,1.37 \pm 0.81,1.57 \pm 1.04$ and $1.63 \pm 0.85$ in 6 weeks, 3 months, 6 months and 1 year after the last injection, respectively. At the same follow-up period for L-PRP group, value of VAS improved from 
$1.93 \pm 1.08$ to $1.67 \pm 0.99,1.43 \pm 1.01,1.60 \pm 1.07$ and $1.77 \pm 0.90$ in 6-week, 12-week, 6-month and 12month follow-up, respectively.

As is shown in Table 2 and Fig. 3, the outcome of VAS motion showed more significant improvement. Value of VAS static in P-PRP group remained stable from the end of the therapy to the 6-month follow-up, whereas they became significantly worse at the 1-year follow-up.

The baseline VAS static and motion scores in P- and L-PRP group were comparable. Although with similar trends, the difference of VAS motion scores in these two groups was significant 6-week follow-up postoperatively.

\section{WOMAC score}

As is shown in Table 2 and Fig. 4, for P-PRP group, WOMAC total improved from $37.37 \pm 13.84$ to $22.83 \pm$ 10.856 weeks after treatment; a further improvement to $20.73 \pm 11.42,19.63 \pm 10.15$ and $31.73 \pm 10.50$ was seen at 3 months, 6 months and 1 year postoperatively. Similar improving tendency was also seen in L-PRP group: WOMAC total improved from $36.23 \pm 12.56$ to $25.67 \pm 13.32,22.30 \pm 11.02,19.97 \pm 9.74$ and $32.17 \pm 10.82$, 6 weeks, 3 months, 6 months and 1 year postoperatively, respectively.

When comparing the two groups, no statistically significant difference was found in WOMAC total before and at any follow-up time after the treatment. However, 6 weeks after treatment, WOMAC pain of P-PRP group was significantly better than L-PRP $(p<0.05)$.

\section{Safety and adverse events}

A total of 15 complications were presented in this study. Mild swelling and local pain occurred in 4 and 8 patients in P-PRP and L-PRP group, respectively. Serious swelling and mild fever not beyond $37.5^{\circ} \mathrm{C}$ occurred in 3 patients in L-PRP group. The fever of one febrile patient in the L-PRP group last for 1 week until a further arthroscopic debridement was applied. The other two febrile patients relieved within 3 days without special treatment. All the other related patients reported symptoms relieved in 1 to 3 days, and no residual symptoms presented. There were no severe complications, including infection, were found in the P-PRP group.

\section{Discussion}

As a carrier of multiple growth factors, PRP is widely used in the treatment of cartilage lesions in the early stages $[15,25,33]$. To date, the results of previous research and our study have shown a positive clinical efficacy of PRP in inhibiting inflammatory reactions, alleviating pain, and promoting the repair of cartilage lesions [21,34]. However, because of the difference in the preparation technology of PRP, PRP with diverse components, especially the concentration of leukocytes, may lead to adverse effects in the treatment of cartilage lesions. [21]

In this study, the short-term results demonstrated a significant difference between the clinical efficacy of P-PRP and L-PRP in treating knee cartilage lesions. The VAS and WOMAC scores in the two groups 
remained stable from the end of the therapy to the 6-month follow-up, but they became significantly worse at the 1-year follow-up. According to previous studies, such a low score at the end of the therapy was often explained by the low patient activity level, rather than from any persistent knee pain or functional limitation [35]. On the other hand, no statistically significant difference was found in WOMAC total, while the WOMAC pain score in the P-PRP group was significantly better than that in the L-PRP group 6 weeks after treatment. With respect to adverse events, the P-PRP group showed better safety and fewer complications, which coincided with those reported in other studies [36].

Once injured, the cartilage has a very limited intrinsic healing capacity. With fewer vessels, nerves, and lymphoid tissue around, cartilage lesions undergo repair only with limited growth factors and stem cells [37]. On the other hand, a rather part of cartilage lesions is attributed to knee degeneration, which is a multifactorial and irreversible disease. PRP, with various high concentrations of growth factors, including transforming growth factor $\beta$, platelet-derived growth factor, insulin-like growth factor, basic fibroblast growth factor, vascular endothelial growth factor, and epidermal growth factor, can promote the proliferation of chondrocytes and the secretion of cartilage matrix, thus inducing the regeneration of cartilage. Moreover, the anti-inflammatory ingredients in PRP help to protect chondrocytes. $[16,20,25,29$, $31,38,39]$

Despite the benefit of PRP in treating cartilage lesions owing to the abundant growth factors, the clinical efficacy of PRP remains a controversial issue, mostly attributed to the different concentrations of leukocytes in PRP. In recent years, several subsequent studies have reported that despite its positive antiinfection effects, L-PRP may lead to the inhibition of the repair of cartilage lesions due to its high concentration of leukocytes. Similar to the results of previous studies, our study recorded more adverse events in the L-PRP group, with mild swelling and local pain. Among them, the fever of one febrile patient in the L-PRP group lasted for 1 week until further arthroscopic debridement was performed.

Inflammatory factors, mainly interleukin-1 $\beta$ (IL-1 $\beta$ ) and tumor necrosis factor a (TNF- $\alpha)$, play a key role in the development of osteoarthritis (OA). Sundman et al. [39] first reported the influence of cellular composition on the growth factor and catabolic cytokine concentrations of PRP. In their study, it was found that platelets increased anabolic signaling and in contrast, leukocytes increased catabolic signaling molecules. Cavallo et al. [26] demonstrated distinct effects on human articular chondrocytes induced by L-PRP and P-PRP in vitro. They found that P-PRP stimulated chondrocyte anabolism by the expression of type-II collagen and aggrecan, whereas L-PRP promoted catabolic pathways involving various cytokines. The findings of Rios et al. [38] suggested that anabolic and anti-inflammatory joint responses depend on the leukocyte and platelet concentrations of PRP preparation. Moreover, P-PRP is recommended to be more effective for the medical treatment of patients with $O A$ and inflammatory synovitis. Despite several RCTs on this topic, L-PRP and P-PRP were directly compared in only a single trial, while they were each compared with common references (hyaluronic acid or placebo) in multiple trials [33]. 
Considering the controversial clinical efficacy of PRP with different concentrations of leukocytes, we decided to conduct an RCT with a larger sample size, a longer follow-up period, and higher evidence. Different extracting approaches to acquire P-PRP and L-PRP were also applied in the study. Presently, as various preparation systems have been used to acquire PRP, there can be a huge difference in the concentration of growth factors and leukocytes in PPR. Bausset et al. [40] evaluated the effect of different centrifugation speeds and time storage durations on platelet quantity and quality.

Approximately 130 and $250 \mathrm{~g}$ successive speed centrifugations were recommended to obtain a highly concentrated and pure PRP product. However, a previous study showed unsatisfactory retrieval rates of platelets by such a method [36]. In our study, two centrifugations (the first at 1,800 rpm for $15 \mathrm{~min}$ to separate erythrocytes and a second at 3,500 rpm for $10 \mathrm{~min}$ to concentrate platelets and separate leukocytes) were applied to produce PRP. For convenience, we chose to make the same volume for the two types of PRP. Considering the difference in extracting methods between P-PRP and L-PRP, the concentration of platelets and leukocytes in L-PRP was higher than that in P-PRP, which may also be the reason for a higher number of early-stage adverse events in the L-PRP group and similar long-term clinical efficacy between the two groups. As there are more adverse events (including a case with persistent fever) with L-PRP, P-PRP is recommended for safety.

Although our study showed better early clinical efficacy and safety of P-PRP in treating knee cartilage lesions compared with those of L-PRP, no significant difference was observed in the long-term follow-up. More RCTs with larger sample sizes and longer follow-up periods are needed. There are also several obstacles to overcome, such as improving methods to extract and purify the product and the selective activation of various components of PRP.

Our study had some limitations. First, the PRP injection was limited and not equal among patients. Second, the sample size was small, and the follow-up period was short. Restricted to the will of patients, no further blank controls were conducted. Finally, we were unable to perform either a routine second-look arthroscopy or an MRI. In addition, we lost nearly $8 \%$ of our patients when they refused to participate in follow-up visits.

\section{Conclusion}

The short-term results of our study are encouraging and demonstrate that both L-PRP and P-PRP intraarticular injections reduce pain and improve function in patients with knee cartilage lesions. Compared with the L-PRP injection, the P-PRP group (which had a lower risk of early inflammation caused by leukocytes) showed better clinical efficacy in the early phase of postoperative rehabilitation and resulted in fewer adverse events. However, long-term clinical efficacy for both injections were similar and weakened after 12 months.

\section{Declarations}

\section{Ethics approval and consent to participate}


The study conformed to the principles of the Declaration of Helsinki. The trial was endorsed by the Shanghai Changzheng Hospital Ethics Committee (CZEC (2017)-04), and written informed consent was obtained. The trial was registered (ChiCTR1900026365).

\section{Consent for publication}

Written informed consent for publication of their clinical details and/or clinical images were obtained from the patient/parent/guardian/relative of the patient.

\section{Availability of data and materials}

The datasets generated and/or analyzed during the current study are not publicly available because it contains patients' personal information but are available from the corresponding author on reasonable request.

\section{Competing interests}

The authors declare that they have no competing interests.

\section{Funding}

This study was supported by the National Natural Science Foundation of China (grant number: 81972087) and Chinese PLA General Hospital (grant number: CXPY201812 and QNF19009).

\section{Authors' contributions}

Q.Q., X.Y. and S.W. equally contributed to the overall design of the project, execution of the study methods, construction of the manuscript, and approval of the submitted and final versions, and should be considered as co-corresponding authors. Y.Z., H.L. and S.C. equally contributed to the execution of the study methods and construction of the manuscript and should be considered as co-first authors. J.S., Q.F., Y.H., B.W., J.W., D.X., Z.L., H.W., and J.Z. oversaw the project overall, contributed to the execution of the study methods and data collections. All authors have read and approved the final submitted manuscript.

\section{References}

1. Paatela T, et al. Assessment of Cartilage Repair Quality With the International Cartilage Repair Society Score and the Oswestry Arthroscopy Score. J Orthop Res. 2020;38(3):555-62.

2. Piasecki DP, et al. Intraarticular injuries associated with anterior cruciate ligament tear: findings at ligament reconstruction in high school and recreational athletes. An analysis of sex-based differences. Am J Sports Med. 2003;31(4):601-5.

3. Schmal H, et al., Comparison of Regenerative Tissue Quality following Matrix-Associated Cell Implantation Using Amplified Chondrocytes Compared to Synovium-Derived Stem Cells in a Rabbit 
Model for Cartilage Lesions. Stem Cells Int, 2018. 2018: p. 4142031.

4. Zhou Y, et al. The clinical efficacy of arthroscopic therapy with knee infrapatellar fat pad cell concentrates in treating knee cartilage lesion: a prospective, randomized, and controlled study. J Orthop Surg Res. 2021;16(1):87.

5. Bannuru RR, et al. Relative efficacy of hyaluronic acid in comparison with NSAIDs for knee osteoarthritis: a systematic review and meta-analysis. Semin Arthritis Rheum. 2014;43(5):593-9.

6. Bowman S, et al. Recent advances in hyaluronic acid based therapy for osteoarthritis. Clin Transl Med. 2018;7(1):6.

7. Han C, et al. The effect of distal tibial tuberosity high tibial osteotomy on postoperative patellar height and patellofemoral joint degeneration. J Orthop Surg Res. 2020;15(1):466.

8. Chen W, et al. Study on the potential active components and molecular mechanism of Xiao Huoluo Pills in the treatment of cartilage degeneration of knee osteoarthritis based on bioinformatics analysis and molecular docking technology. J Orthop Surg Res. 2021;16(1):460.

9. Huang $Y$, et al. Intra-articular injections of platelet-rich plasma, hyaluronic acid or corticosteroids for knee osteoarthritis: A prospective randomized controlled study. Orthopade. 2019;48(3):239-47.

10. Biant LC, et al. The surgical management of symptomatic articular cartilage defects of the knee: Consensus statements from United Kingdom knee surgeons. Knee. 2015;22(5):446-9.

11. Kon E, et al. Platelet-rich plasma (PRP) to treat sports injuries: evidence to support its use. Knee Surg Sports Traumatol Arthrosc. 2011;19(4):516-27.

12. Jia J, et al., The Differential Effects of Leukocyte-Containing and Pure Platelet-Rich Plasma on Nucleus Pulposus-Derived Mesenchymal Stem Cells: Implications for the Clinical Treatment of Intervertebral Disc Degeneration. Stem Cells Int, 2018. 2018: p. 7162084.

13. Fei $\mathrm{X}$, et al., Platelet-rich plasma has better mid-term clinical results than traditional steroid injection for plantar fasciitis: A systematic review and meta-analysis. Orthop Traumatol Surg Res, 2021: p. 103007.

14. Sampson $S$, et al. Injection of platelet-rich plasma in patients with primary and secondary knee osteoarthritis: a pilot study. Am J Phys Med Rehabil. 2010;89(12):961-9.

15. Filardo G, et al. Platelet-rich plasma intra-articular knee injections for the treatment of degenerative cartilage lesions and osteoarthritis. Knee Surg Sports Traumatol Arthrosc. 2011;19(4):528-35.

16. Ba R, et al., Increased Expression of Sox9 during Balance of BMSCs/Chondrocyte Bricks in PlateletRich Plasma Promotes Construction of a Stable 3-D Chondrogenesis Microenvironment for BMSCs. Stem Cells Int, 2020. 2020: p. 5492059.

17. Kennedy MI, et al. Platelet-Rich Plasma and Cartilage Repair. Curr Rev Musculoskelet Med. 2018;11(4):573-82.

18. Rodas G, et al. Autologous bone marrow expanded mesenchymal stem cells in patellar tendinopathy: protocol for a phase I/II, single-centre, randomized with active control PRP, double-blinded clinical trial. J Orthop Surg Res. 2019;14(1):441. 
19. Tang JZ, et al. Platelet-rich plasma versus hyaluronic acid in the treatment of knee osteoarthritis: a meta-analysis. J Orthop Surg Res. 2020;15(1):403.

20. Sanchez M, et al. Intra-articular injection of an autologous preparation rich in growth factors for the treatment of knee OA: a retrospective cohort study. Clin Exp Rheumatol. 2008;26(5):910-3.

21. Zhou $Y$, et al. The differential effects of leukocyte-containing and pure platelet-rich plasma (PRP) on tendon stem/progenitor cells - implications of PRP application for the clinical treatment of tendon injuries. Stem Cell Res Ther. 2015;6:173.

22. Dohan Ehrenfest DM, Rasmusson L, Albrektsson T. Classification of platelet concentrates: from pure platelet-rich plasma (P-PRP) to leucocyte- and platelet-rich fibrin (L-PRF). Trends Biotechnol. 2009;27(3):158-67.

23. Saklatvala J. Tumour necrosis factor alpha stimulates resorption and inhibits synthesis of proteoglycan in cartilage. Nature. 1986;322(6079):547-9.

24. Burguera EF, et al. Effect of hydrogen sulfide sources on inflammation and catabolic markers on interleukin 1 beta-stimulated human articular chondrocytes. Osteoarthritis Cartilage. 2014;22(7):1026-35.

25. Castillo TN, et al. Comparison of growth factor and platelet concentration from commercial plateletrich plasma separation systems. Am J Sports Med. 2011;39(2):266-71.

26. Cavallo $\mathrm{C}$, et al. Comparison of platelet-rich plasma formulations for cartilage healing: an in vitro study. J Bone Joint Surg Am. 2014;96(5):423-9.

27. Lana JF, et al. Leukocyte-rich PRP for knee osteoarthritis: Current concepts. J Clin Orthop Trauma. 2019;10(Suppl 1):S179-82.

28. Moojen DJ, et al. Antimicrobial activity of platelet-leukocyte gel against Staphylococcus aureus. J Orthop Res. 2008;26(3):404-10.

29. Bielecki TM, et al. Antibacterial effect of autologous platelet gel enriched with growth factors and other active substances: an in vitro study. J Bone Joint Surg Br. 2007;89(3):417-20.

30. Bryan N, et al. Reactive oxygen species (ROS)-a family of fate deciding molecules pivotal in constructive inflammation and wound healing. Eur Cell Mater. 2012;24:249-65.

31. Blotnick S, et al. T lymphocytes synthesize and export heparin-binding epidermal growth factor-like growth factor and basic fibroblast growth factor, mitogens for vascular cells and fibroblasts: differential production and release by CD $4+$ and CD $8+T$ cells. Proc Natl Acad Sci U S A. 1994;91(8):2890-94.

32. Riboh JC, et al. Effect of Leukocyte Concentration on the Efficacy of Platelet-Rich Plasma in the Treatment of Knee Osteoarthritis. Am J Sports Med. 2016;44(3):792-800.

33. Filardo $\mathrm{G}$, et al. Platelet-rich plasma intra-articular injections for cartilage degeneration and osteoarthritis: single- versus double-spinning approach. Knee Surg Sports Traumatol Arthrosc. 2012;20(10):2082-91. 
34. Zhou Y, Wang JH, PRP Treatment Efficacy for Tendinopathy: A Review of Basic Science Studies. Biomed Res Int, 2016. 2016: p. 9103792.

35. Kon E, et al. Platelet-rich plasma: intra-articular knee injections produced favorable results on degenerative cartilage lesions. Knee Surg Sports Traumatol Arthrosc. 2010;18(4):472-9.

36. Magalon $\mathrm{J}$, et al. Characterization and comparison of 5 platelet-rich plasma preparations in a singledonor model. Arthroscopy. 2014;30(5):629-38.

37. Oldershaw RA. Cell sources for the regeneration of articular cartilage: the past, the horizon and the future. Int J Exp Pathol. 2012;93(6):389-400.

38. Rios DL, et al. Effects over time of two platelet gel supernatants on growth factor, cytokine and hyaluronan concentrations in normal synovial membrane explants challenged with lipopolysaccharide. BMC Musculoskelet Disord. 2015;16:153.

39. Sundman EA, Cole BJ, Fortier LA. Growth factor and catabolic cytokine concentrations are influenced by the cellular composition of platelet-rich plasma. Am J Sports Med. 2011;39(10):2135-40.

40. Bausset $\mathrm{O}$, et al. Formulation and storage of platelet-rich plasma homemade product. Biores Open Access. 2012;1(3):115-23.

\section{Figures}



\section{Figure 1}

The process to prepare PRP. 1a is the product after the first centrifugation to separate erythrocytes and WBC. $1 \mathrm{~b}$ is the product to acquire L-PRP after the second centrifugation at 3,500 rpm for $10 \mathrm{~min}$ to concentrate platelets. $1 \mathrm{c}$ is the product to acquire L-PRP after the second centrifugation at 3,500 rpm for 10 min to concentrate platelets. 


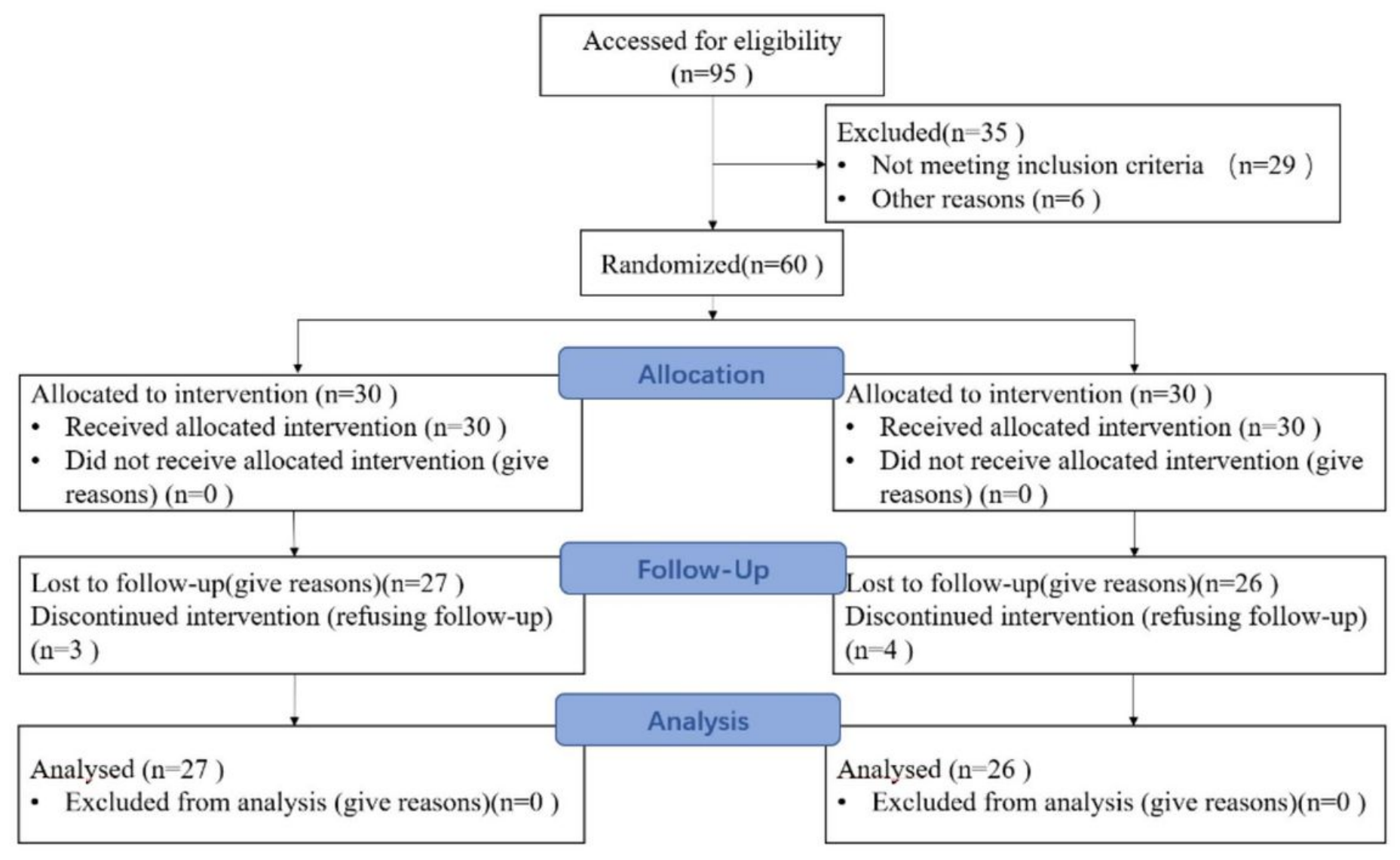

Figure 2

The CONSORT flowchart diagram of the whole process.

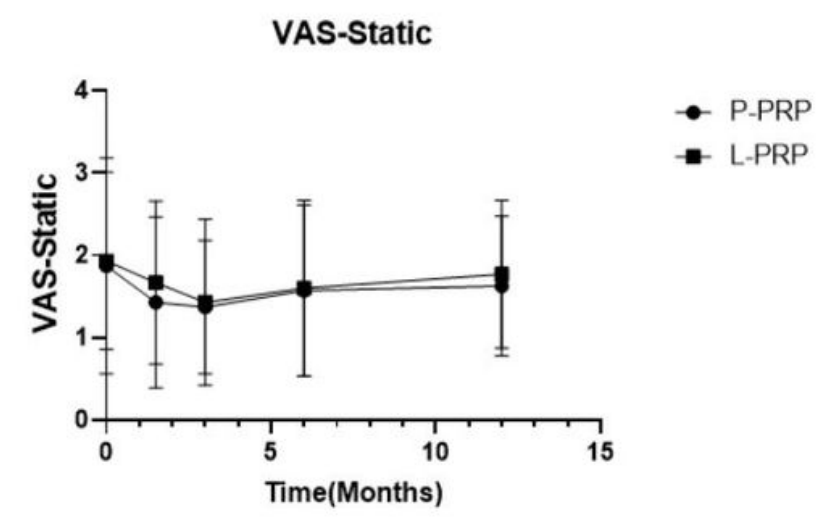

$\mathbf{a}$

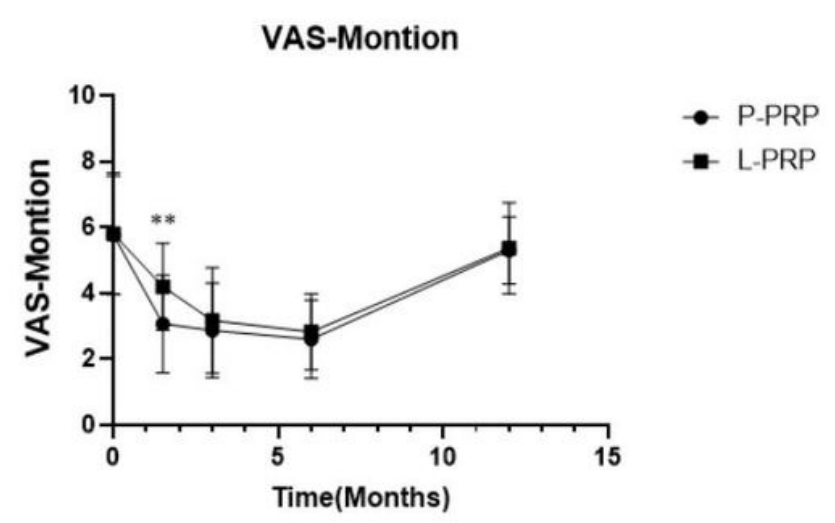

b

\section{Figure 3}


VAS scores were evaluated pre-operation, 6 weeks, 12 weeks, 6 months, 12 months after intervention. a: VAS static scores; b: VAS motion scores $(\forall \mathbb{D} p<0.01)$

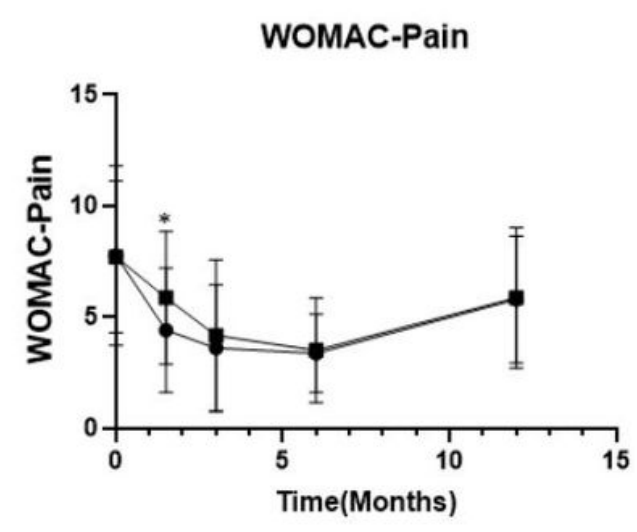

a

WOMAC-Function

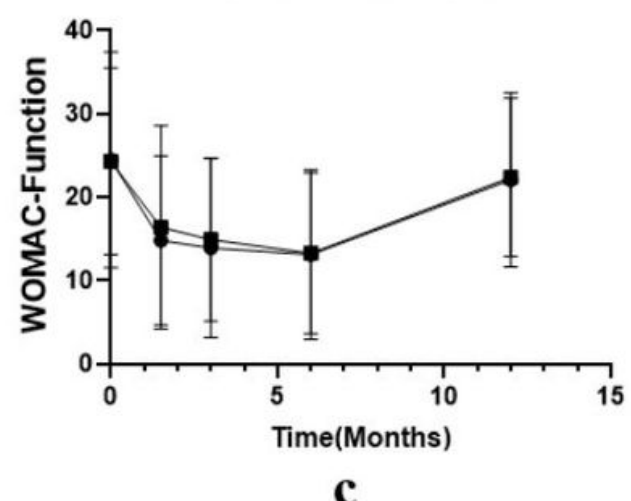

WOMAC-Stiffness

$\bullet$ P-PRP

- L-PRP

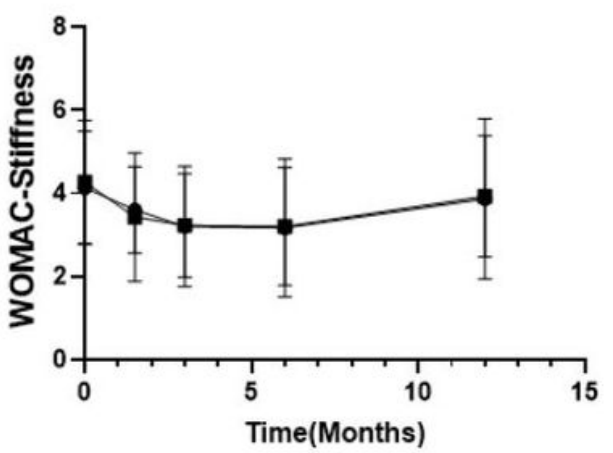

b

WOMAC-Total

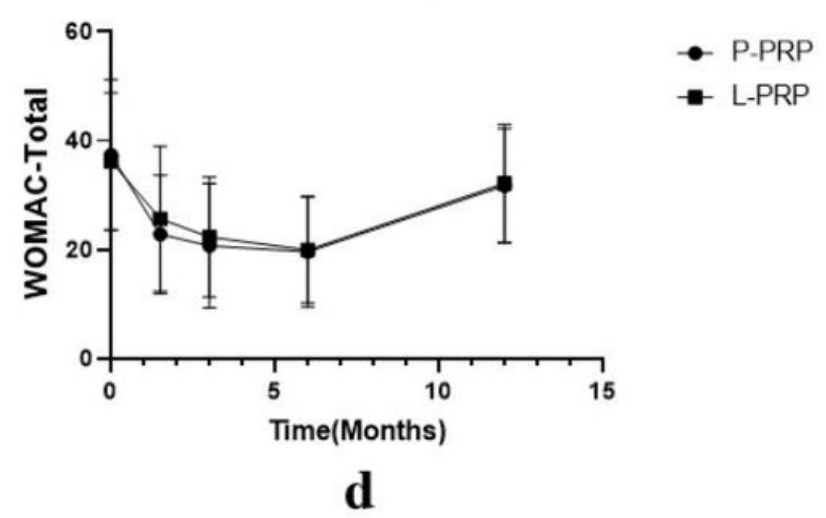

Figure 4

WOMAC scores were evaluated pre-operation, 6 weeks, 12 weeks, 6 months, 12 months. a: WOMAC pain

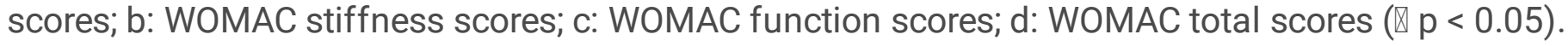

